\title{
What a long, strange trip it's been
}

Launched in 1977, NASA's Voyager missions transformed humanity's view of the Solar System. Now in their fourth decade, they are sending back information about the borderlands of interstellar space (see pages 63-83). Here, three veterans recall details and moments that meant something special along the way.

\section{The little motor that could}

We needed an instrument that scanned the sky in order to determine the direction that particles flying past the spacecraft were travelling in. But how do you get an instrument to scan $360^{\circ}$ of the sky when the spacecraft on which it's mounted doesn't rotate, and must always point to Earth?

Our answer was to mount the instrument on a small platform and have an electrical stepping motor move it $45^{\circ}$ every minute or two - sometimes a lot faster. The engineers, being a conservative bunch, thought that after a few thousand steps the motor would seize up. Remember, it was 1971! A design that involved moving parts on a spacecraft was not considered a good idea - the initial response was 'Are you crazy?'. I insisted, and we tested the motor for about 500,000 steps, which was twice what we thought we'd need for the trip to Saturn.

Over 30 years on and after well over 5 million steps the little motor is still stepping dutifully once every 192 seconds. That's 10 million steps if we count Voyagers 1 and 2. Not only did we get wonderful directional data as we went through the magnetospheres of four planets, but the direction of particle flow signalled the approach to the termination shock a couple of years before we actually got there!

It was the riskiest decision I ever made, but it paid off big time, scientifically. For a scientist, the Voyager mission is the stuff that dreams are made of.

Stamatios Krimigis has been principal investigator of the low-energy charged particle experiments since 1971.

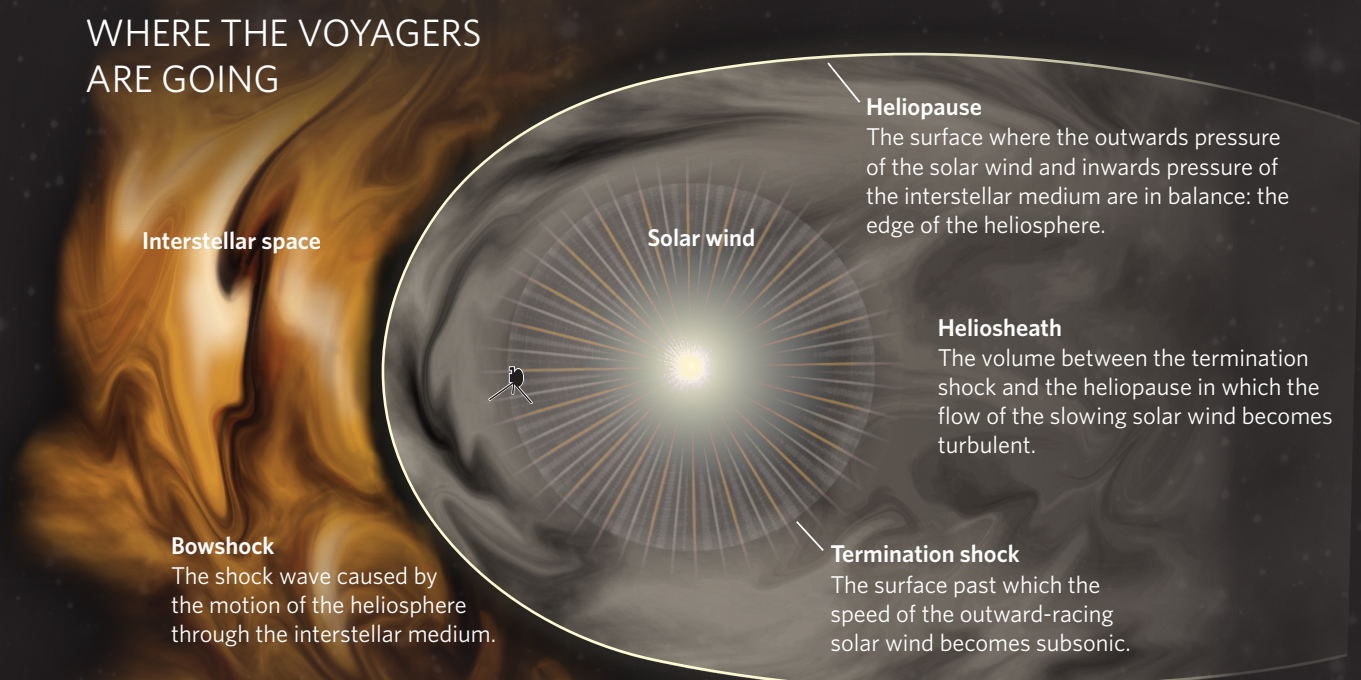


For more information on the Voyager missions, see the News and Views article on page 38; the sequence of six Letters starting on page 63; the video at www.nature. com/nature/videoarchive/voyager; and the podcast at http://www. nature.com/nature/podcast.

\section{The cup that cheers}

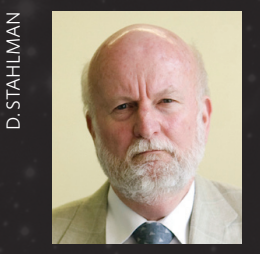

The instrument concept for the Plasma spectrometer - the 'Faraday cup' design - dates from the beginning of the space age. The late Herb Bridge, my mentor at the Massachusetts Institute of Technology, used something very similar to make the first measurements of the energy spectrum of the shocked solar wind in the earth's magnetosheath in 1961, when he was principal investigator on the plasma instrument on Explorer 10.

And there's an excellent chance that, before I retire, the Faraday cup on Voyager will directly measure the plasma in the interstellar medium. I was in high school when Explorer 10 went up, and in my lifetime we have gone from being just above the atmosphere to reaching for the interstellar medium. Pretty nifty.

Everyone knows about the golden records on the Voyagers that Carl Sagan worked on. Our cup collector has a message too. The names of all the people at MIT who worked on the plasma probe are written on the surface underneath the motto "Live Free or Die". Our chief engineer on the project was Bob Butler, a New Hampshire resident, and that's the New Hampshire state motto. John Belcher was principal investigator of the plasma spectrometer from 1986 to 1990.

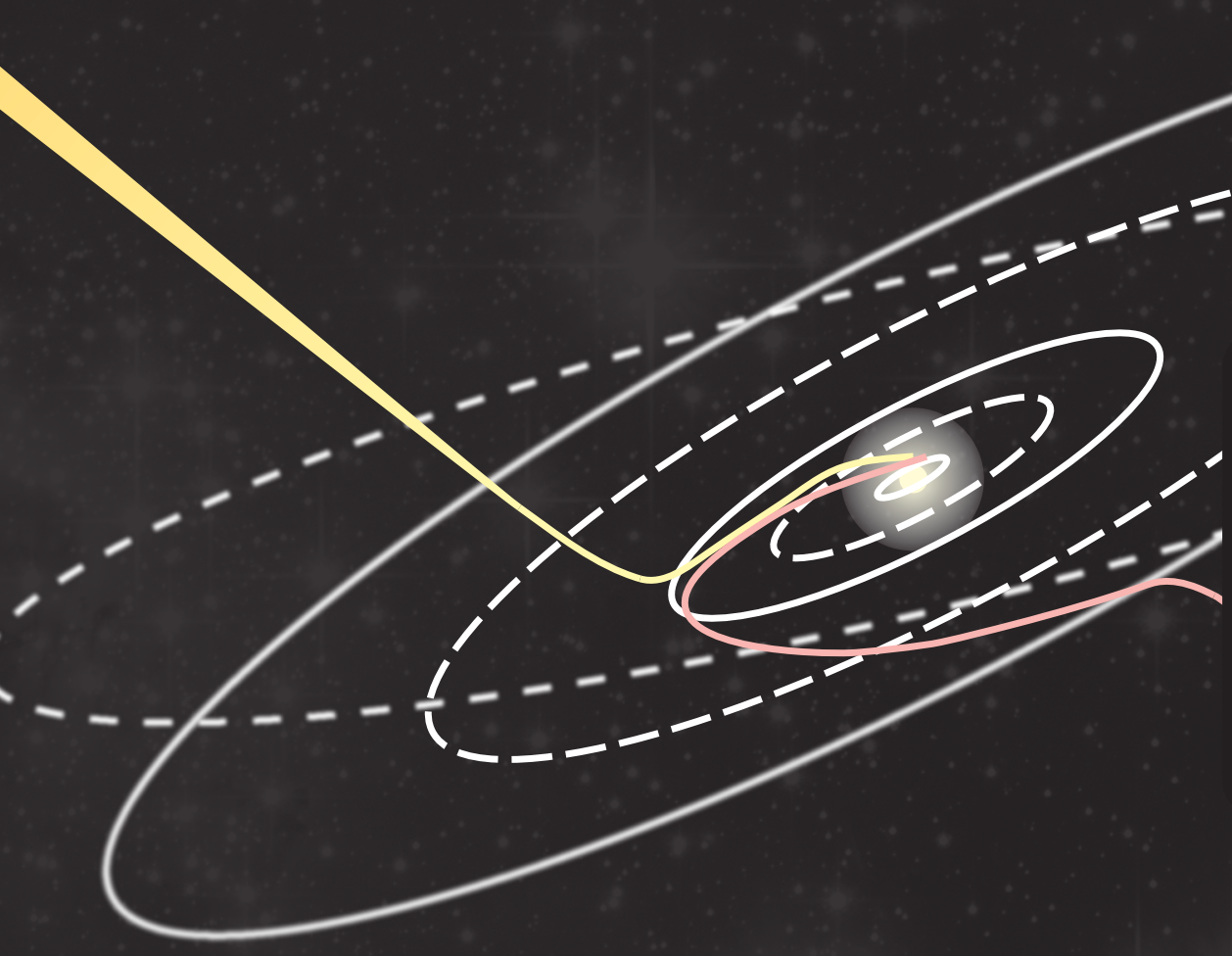

\section{The mission of a lifetime}

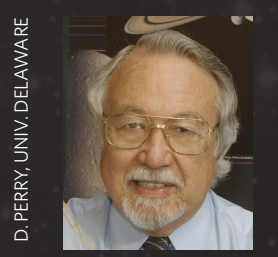

The most exciting moments for me were the six close planetary encounters, especially Voyager 2's discovery of previously unknown and unpredicted but significant intrinsic global magnetic fields at Uranus and Neptune, with their entrapped radiation belts. It was surely as exciting as discovering the magnetic field of Mercury in 1974 and 1975 with Mariner 10, or discovering the complexly magnetized crust of Mars in 1999 with the orbiting Mars Global Surveyor.

For Voyager 2's Uranus fly-by in 1986 the magnetometer team invited James Van
Allen, discoverer of Earth's radiation belts in 1958, to join us at the Jet Propulsion Laboratory for the encounter activities. He was as delighted to participate in the real-time learning episode as if he were a fellow principal investigator.

Over the years the magnetometer team has been downsized by $50 \%$, mainly by recent retirements. But along with longtime NASA Goddard Space Flight Center colleagues Mario Acuña and Leonard Burlaga we soldier on as the data continue to flow in. It continues to be the mission of a lifetime.

Norman Ness has been principal investigator of the magnetometers since 1971. 